\title{
Natural killer cell immunotherapy: from bench to bedside
}

\author{
Anna Domogala ${ }^{1,2}$, J. Alejandro Madrigal ${ }^{1,2}$ and Aurore Saudemont ${ }^{1,2 *}$ \\ ${ }^{1}$ Anthony Nolan Research Institute, London, UK, ${ }^{2}$ University College London, London, UK
}

The potential of natural killer (NK) cells to target numerous malignancies in vitro has been well documented; however, only limited success has been seen in the clinic. Although NK cells prove non-toxic and safe regardless of the cell numbers injected, there is often little persistence and expansion observed in a patient, which is vital for mounting an effective cellular response. NK cells can be isolated directly from peripheral blood, umbilical cord blood, or bone marrow, expanded in vitro using cytokines or differentiated in vitro from hematopoietic stem cells. Drugs that support NK cell function such as lenalidomide and bortezomib have also been studied in the clinic, however, the optimum combination, which can vary among different malignancies, is yet to be identified. NK cell proliferation, persistence, and function can further be improved by various activation techniques such as priming and cytokine addition though whether stimulation pre- or post-injection is more favorable is another obstacle to be tackled. Here, we review the various methods of obtaining and activating NK cells for use in the clinic while considering the ideal product and drug complement for the most successful cellular therapy.

Keywords: natural killer cells, cancer, immunotherapy, activation, proliferation, persistence

\section{Introduction}

Natural killer (NK) cells are unique lymphocytes, distinct from B and T cells, which bridge the innate and adaptive immune systems. They have the unique capacity to exert immunoregulatory and cytotoxic functions against transformed and infected cells without prior sensitization. NK cells are characterized by the expression of CD56 and absence of CD3 and can be further subdivided into a CD56 $6^{\text {bright }}$ population, which is predominantly cytokine producing and a CD56 ${ }^{\mathrm{dim}}$ population, which is cytolytic and provides antibody-dependent cell-mediated cytotoxicity via CD16 (1). NK cells operate by detecting information, which is missing on the target. This phenomenon is known as the "missing self hypothesis" and postulates that NK cell cytotoxicity inversely correlates with the target expression of major histocompatibility complex class I (MHCI) $(2,3)$. In addition, NK cell activity is further regulated by a complex array of inhibitory and activating receptors such as killer cell immunoglobulin-like receptors (KIR), natural cytotoxicity receptors (NKp44, NKp30, and NKp46), and C-type lectins (CD94/NKG2A/NKG2C/NKG2D). These properties equip NK cells with the tools to actively eliminate susceptible targets (4). Taking into account, the cytotoxic potential of these cells numerous attempts have been made to transfer NK cell immunotherapy into the clinic. Here, we review which methods to consider for obtaining cells for therapy, drug complements, and pre-infusion activation techniques. We also summarize current clinical trials and outcomes and postulate where success in NK immunotherapy may lie. 


\section{NK Cells and Cancer}

Natural killer cells were first implicated as playing a role in cancer immunosurveillance when one large epidemiologic study found that low NK cell cytotoxicity forecasted an increased risk in developing cancer (5). There have since been numerous studies, which demonstrate that NK cells can target human tumors in vivo making them a desirable candidate for therapeutic use (6). Clinical trials using autologous NK cells have shown the therapy to be nontoxic, however, they fail to prove efficacy (7), which could be the result of inhibition by self-MHC-I. Allogeneic treatment therefore has potential to offer an alternative therapy with improved effect. The direct involvement of allo-reactive NK cells in inducing antitumor effect in hematopoietic transplants was first demonstrated in 2002 (8). NK cells showed to enhance engraftment; providing graft vs. leukemia (GvL) effect while suppressing graft vs. host disease (GvHD) particularly when a KIR ligand mismatch in the donor to host direction was observed. Reduced GvHD was hypothesized to be attributed to the lysis of the recipient's antigen presenting cells (APCs) reducing the incidence of GvHD while maintaining GvL effect. This was later successfully translated into an in vivo model using acute myeloid leukemia (AML)-engrafted NOD/SCID mice infused with allo-reactive NK cells. Tumor clearance was achieved implicating NK cells in preserving the GvL effect (9).

Miller and colleagues later translated NK cell therapy alone into the clinic where allogeneic NK cells were infused into patients with advanced cancer alongside IL-2 administration. This demonstrated that NK cell infusions were feasible and safe and led to complete remission in 5/19 patients with poor prognosis AML (10). Additionally, the efficacy of haploidentical NK cell therapy in the refractory disease was further improved by depleting host regulatory $\mathrm{T}$ cells with IL-2 diphtheria toxin preventing their immunosuppressive effect (11). NK cell allo-reactivity could also be utilized in other scenarios besides hematopoietic stem cell transplantation (HSCT) with studies in malignant glioma and neuroblastoma patients demonstrating that NK cell infusions are safe and partially effective $(12,13)$. Numerous types of cancer could therefore benefit from NK cell immunotherapy and current clinical trials include pancreas, lungs, head/neck, breast, and renal cell carcinomas.

\section{Clinical Conditioning}

Not only chemotherapy and/or radiotherapy are required for the success of HSCT but also cellular immunotherapy. Such treatments are necessary to reduce tumor burden and suppress the immune system of the patient to prevent rejection of the cellular therapy. Defining the correct conditioning regimen is therefore critical. In a transplantation setting, common regimens are referred to as myeloablative, non-myeloablative, and reduced intensity and their use will depend on patient age and disease severity; however, any decrease of leukemia recurrence is often at the expense of an increase in toxicity (14).

The use of new conditioning agents termed as "novel agents" have become increasing popular in cancer immunotherapy as a result of their immunomodulatory and direct tumor targeting mechanisms. In combination with cellular therapy, they offer the potential for a more personalized and less toxic treatment regimen as these specialized drugs have been shown to not only reduce tumor burden but also enhance the function of cellular therapies. Although chemotherapy has revolutionized the treatment of cancer, its side effects include the development of refractory disease and severe toxicity. Novel agents provide an alternative option of harnessing the immune system to tackle malignancies.

Thalidomide was one of the first novel agents to be well studied; it is a synthetic glutamic acid derivative that is capable of immunomodulatory, anti-inflammatory, and anti-angiogenic effects. Although proven successful in targeting multiple myeloma the exact mechanism of action of thalidomide is yet to be elucidated although anti-inflammatory effects have been attributed to inhibition of TNF- $\alpha$ production by monocytes and antiproliferative capabilities to disruption of the bone marrow (BM) microenvironment preventing multiple myeloma cellular development (15). Although extended anti-angiogenic characteristics make a desirable option in limiting tumor development its immunomodulatory properties have not been so well defined. Lenalidomide is an immunomodulatory compound with a dual mechanism of action. It is capable of targeting the tumor directly through stromal support disruption, induction of tumor suppressor genes, and activation of caspases (16). It is also able to stimulate the cytotoxic functions of NK cells and T lymphocytes while limiting the immunosuppressive impact of regulatory $\mathrm{T}$ cells (17). Additionally, bortezomib is a proteasome inhibitor proven popular by up-regulating expression of TRAIL death receptors and altering caspase- 8 activity rendering tumors susceptible to NK cell lysis. However, intriguingly these tumors became resistance to $\mathrm{T}$ cell cytotoxicity (18). The specific mechanisms by which novel agent function offer a promising future for the treatment of a variety of malignancies as these agents target not only the tumor themselves but also offer potential to enhance the immune system. This provides the possibility of coupling cellular therapy with novel agents to provide personalized treatment regimens to target an individual's condition.

\section{NK Cell Sources}

It is considered that the success of NK cell immunotherapy is dependent on obtaining high numbers of functional NK cells that have the potential to survive in vivo. Numerous attempts have therefore been made to obtain high levels of NK cells from a variety of sources. One option is to isolate cells directly from peripheral blood (PB) or cord blood (CB), however, as NK cells make up only $10 \%$ of circulating lymphocytes in $\mathrm{PB}$ and $20 \%$ in CB the number of cells obtained can be limited and could potentially prevent the option for multiple infusions. Doses of $1-2 \times 10^{7}$ cells $/ \mathrm{kg}$ have been identified as safe (19); however, higher doses of $2 \times 10^{8} / \mathrm{kg}$ have been shown to be well tolerated and non-toxic (20). Several techniques have therefore been explored to increase cell numbers. This includes expanding isolated cells in vitro using different combinations of cytokines with or without feeder layers, the use of NK cell lines and differentiating NK cells from hematopoietic stem cells (HSCs). 


\section{NK Cell Expansion}

Numerous methods of expanding mature NK cells in vitro have been explored and have been reviewed previously (21); however, these products seem to produce limited clinical success. This may be because of wide variations in expansion rate and distribution of NK cell subpopulations (22) or expanding mature cells produces effectors with a more finite lifespan unable to proliferate with lower cytotoxicity post infusion (23). NK cell expansion using aAPCs particularly the GMP-compatible genetically modified form of the K562 myeloid leukemia cell line engineered to express membrane-bound interleukin 15 and the ligand for the co-stimulatory molecule $4-1 \mathrm{BBL}$ has been rising in popularity due to the potential to rapidly expand an NK cell product with an up-regulation of activating receptors and improved killing capacity (24). However, a first-in-human trial carried out by Shah and colleagues in 2014 performing the adoptive transfer of donor-derived IL-15/4-1BBL activated NK cells showed interesting results. Surprisingly, 5/9 patients experienced acute GvHD and as the T cell content of the infusion was well below the specified threshold for GvHD development the group concluded that the aNK-DLI contributed to the effect by stimulating underlying $\mathrm{T}$ cell allo-reactivity (25). This is the first time in a clinical setting NK cells have been implicated in the role of induction or aggravation of $\mathrm{GvHD}$, which could be a result of lack of immunosuppressive drugs post transplant or infusion of IL-2, which expands immunoregulatory populations. This coupled with the infusion of an expanded NK cell population with such a high up-regulation of activating receptors could be the reason for such unfavorable results.

\section{NK Cell Lines}

The use of NK cell lines have been seen as an attractive option due to the availability of a clinical grade frozen stock and their homologous nature. The most prominent NK cell line currently in focus is NK-92, which was established from a patient with non-Hodgkin's lymphoma and has demonstrated the capability of lysing leukemia, lymphoma, and myeloma in vitro (26). Current clinical trials have proven non-toxic; however, they have shown limited success in demonstrating efficacy $(27,28)$. This could be a result of the necessity to irradiate a cell line prior to infusion for safety requirements, the cells could therefore be incapable of proliferation in vivo severely limiting their persistence and potential to target the tumor.

\section{Differentiation of NK Cells}

Differentiating NK cells in vitro from HSCs or induced pluripotent stem (iPS) cells are alternative options for obtaining high numbers of functional cells. Different sources of cryopreserved HSCs have been used to differentiate NK cells in vitro including human embryonic stem cells (hESC), BM, mobilized peripheral blood stem cells (mPBSC), and cord blood stem cells (CBSC). hESC are a controversial source due to the ethical dilemma posed by obtaining cells from a 5- to 7-day-old embryo. However, the H9 hESC cell line has been used to produce NK cells that express activating and inhibitory receptors, including KIRs, and are able to produce cytokines and mediate cytotoxicity in vitro and in vivo (29). The invasive collection procedure limits the use of BM and has therefore mainly been used to study NK cell development (30, 31). Differentiating NK cells from induced pluripotent cells offers potential due to the ready availability of a donor and the non-invasive cell harvesting methods. A recent study identified a method of differentiating mature and functional NK cells using a combination of embryoid body formation and membrane-bound interleukin 21-expressing aAPCs (32) and a thorough review of the potential uses of such cells in the clinic was published last year (33). The possibility of reprograming cells is a promising one; however, there is the possible limitation that the differentiated NK cells will be suppressed by self-MHC and therefore have little cytotoxic effect. The use of NK cells differentiated from $\mathrm{CD} 34^{+}$progenitors was first shown to be feasible in the clinic by Yoon and colleagues in 2010 (34). This led to interest in the use of umbilical cord blood $\mathrm{CD}_{3}{ }^{+}$cells as a source of NK cells with the focus being on generating a readily available, non-invasive, off the shelf cellular product (35). Our group modified a published protocol (36) and compared the use of mPBSC, fresh CBSC, and frozen CBSC at differentiating NK cells in vitro (37). This work demonstrated frozen $\mathrm{CB}$ CD $34^{+}$ cells to be the best source of NK cells over fresh $\mathrm{CB} \mathrm{CD} 34^{+}$and frozen mPBSCs. This was due to higher fold expansion and therefore higher NK cell numbers generated without compromising on phenotype, cytokine production, or cytotoxicity. Additionally, the cells are capable of further proliferation in vitro and more importantly could persist for longer and in higher numbers in vivo. Considering that proliferation and persistence of $\mathrm{NK}$ cells in vivo is fundamental for the development of a clinically relevant cellular product this makes the differentiation of NK cells from CB HSCs in vitro an attractive candidate for NK cell immunotherapy.

\section{NK Cell Activation}

As reviewed in Table 1, there have been many studies that well document the expansion of NK cells in vitro, however, we are yet to obtain a clinically successful product, which proliferates and persists in vivo inducing consistent efficacy. This could be because we are yet to identify the optimum activation method and status of the cells before infusion. As seen in Figure 1, whether the cells should be incubated with cytokines, genetically engineered, differentiated into a "memory-like" phenotype, or primed using NK nonsusceptible cell lines are all options that need to be considered.

Cytokine activation has always been a popular method of stimulating NK cells as it is a well-documented pathway of activation in vivo and different cytokines can give rise to the same signaling patterns while differing in their effects on development, activation, and proliferation. IL-2 stimulates cellular proliferation and enhances cytotoxicity, however, it has been noted that this only affects a small sub-population for an extended period (55). IL-15 significantly improves NK cell survival although it only stimulates minimal expansion (56). Furthermore, the toxic effects of the in vivo administration of cytokines cannot be ignored, IL-2 risks vascular leak syndrome caused by the stimulation of endothelial cells through the IL-2 receptor (57) and preferentially expands T regulatory cells, which mediate immune suppression (58). Studies with IL-15 in non-human primates have only shown transient 


\begin{tabular}{|c|c|c|c|c|c|c|c|c|c|}
\hline $\begin{array}{l}\text { Initial } \\
\text { population }\end{array}$ & $\begin{array}{l}\text { Feeder } \\
\text { cells }\end{array}$ & $\begin{array}{l}\text { Fold expansion } \\
\text { in vitro (purity) }\end{array}$ & $\begin{array}{l}\text { In vitro } \\
\text { cytokine } \\
\text { admin }\end{array}$ & Condition & $\begin{array}{l}\text { Treatment and } \\
\text { in vivo cytokine } \\
\text { admin }\end{array}$ & Dose & $\begin{array}{c}\text { In vivo } \\
\text { expansion }\end{array}$ & $\begin{array}{l}\text { Clinical } \\
\text { outcome }\end{array}$ & Reference \\
\hline \multicolumn{10}{|c|}{ AUTOLOGOUS } \\
\hline $\begin{array}{l}\text { CD3 } \\
\text { depleted } \\
\text { PBMCs }\end{array}$ & $\begin{array}{l}\text { LCL cell line } \\
\text { (LAZ388) }\end{array}$ & $\begin{array}{l}43 \pm 26 \text { in } \\
13-21 \text { days (90\%) }\end{array}$ & IL-2 & MRC & $\begin{array}{l}\text { High dose IL-2 + LANAK } \\
\text { following initial PR to IL-2 } \\
\text { alone }\end{array}$ & $\mathrm{N} / \mathrm{A}$ & $\mathrm{N} / \mathrm{D}$ & $\begin{array}{l}\text { Induced clinical response } \\
15-30 \% \text { patients }\end{array}$ & (38) \\
\hline PBMCs & None & No expansion & IL-2 & $\begin{array}{l}\text { Advanced CRC and } \\
\text { NSCLC }\end{array}$ & $\begin{array}{l}\text { Multiple infusions of NK } \\
\text { cells + IL-2 + Hsp70 } \\
\text { peptide TKD }\end{array}$ & $\begin{array}{l}1-7.5 \times \\
10^{6} / \mathrm{kg}\end{array}$ & Multi-infusion trial & $\begin{array}{l}\text { Well tolerated and safe, no } \\
\text { significant tumor response }\end{array}$ & (39) \\
\hline PBMCs & $\begin{array}{l}\text { Wilms tumor cell } \\
\text { line (HFWT) }\end{array}$ & $\begin{array}{l}113 \text { in } 14 \text { days } \\
(96 \%)\end{array}$ & $\mathbb{I L}-2$ & Malignant glioma & Multiple infusions + IFN- $\beta$ & $\mathrm{N} / \mathrm{A}$ & Multi-infusion trial & $\begin{array}{l}\text { Well tolerated, no toxicity, } 3 \\
\text { PR, } 2 \text { MR, } 4 \text { NC, and } 7 \text { PD }\end{array}$ & (12) \\
\hline PBMCs & $\begin{array}{l}\alpha \text { GalCer-pulsed } \\
\text { autologous } \\
\text { PBMCs }\end{array}$ & $\begin{array}{l}10^{1}-10^{3} 21 \text { days } \\
\text { (70\% viability) }\end{array}$ & IL-2 & $\begin{array}{l}\text { Recurrent or } \\
\text { advanced NSCLC }\end{array}$ & $\begin{array}{l}\text { Infusion of ex vivo } \\
\text { expanded V } \alpha 24 N K T \text { cells }\end{array}$ & $5 \times 10^{7} / \mathrm{m}^{2}$ & $\begin{array}{l}\text { Reduced function in } \\
\text { some patients }\end{array}$ & Well tolerated, no toxicity & $(40)$ \\
\hline $\begin{array}{l}\mathrm{CD}^{-} / \mathrm{CD}^{2} 6^{+} \\
\text {PBMCs }\end{array}$ & $\begin{array}{l}\text { EBV-LCL } \\
\text { (TM-LCL) }\end{array}$ & $\begin{array}{l}53-683 \text { in } 14 \text { days } \\
(99.7 \%)\end{array}$ & $\mathbb{I L}-2$ & $\begin{array}{l}\text { CLL and metastatic } \\
\text { tumors }\end{array}$ & $\begin{array}{l}\text { Infusion of NKs + IL-2 } \\
\text { after PEN/BOR }\end{array}$ & $1 \times 10^{8} / \mathrm{kg}$ & Multi-infusion trial & $\begin{array}{l}\text { Well tolerated and some } \\
\text { pre-clinical evidence of } \\
\text { anti-tumor response }\end{array}$ & (41) \\
\hline $\begin{array}{l}\mathrm{CD}^{-} \\
\text {PBMCs }\end{array}$ & Auto PBMCs & $\begin{array}{l}278-1097 \text { in } \\
21-26 \text { days } \\
(91-97 \%)\end{array}$ & IL-2 & $\begin{array}{l}\text { Metastatic melanoma } \\
\text { and RCC }\end{array}$ & $\begin{array}{l}\text { Infusion of activated } \\
\text { NKs + IL-2 after CY/FLU } \\
\text { regimen }\end{array}$ & $\begin{array}{l}1.88-7.6 \times \\
10^{10} / \mathrm{kg}\end{array}$ & $\begin{array}{l}\text { NK persistence } \\
7 \text { days post infusion }\end{array}$ & No toxicity or clinical response & (42) \\
\hline \multicolumn{10}{|c|}{ AUTOLOGOUS/ALLOGENEIC } \\
\hline $\begin{array}{l}\mathrm{CD}^{+} 6^{+} / \mathrm{CD}^{-} \\
\text {PBMCs }\end{array}$ & $\begin{array}{l}4-1 \mathrm{BBL}^{+} \mathrm{IL}- \\
15 \mathrm{R} \alpha^{+} \\
\text {aAPCs }\end{array}$ & $\begin{array}{l}12-160 \text { in } \\
7-9 \text { days } \\
\text { (68-99\%) }\end{array}$ & IL-2 & MM & $\begin{array}{l}\text { Infusion of NKs + IL-2 } \\
\text { after BOR/CY/FLU }\end{array}$ & $\begin{array}{l}2 \times 10^{7}-2 \times \\
10^{8} / \mathrm{kg}\end{array}$ & $\begin{array}{l}\text { Significant in vivo } \\
\text { expansion fresh } \\
\text { product }\end{array}$ & Well tolerated, no toxicity & (43) \\
\hline \multicolumn{10}{|l|}{ ALLOGENEIC } \\
\hline $\begin{array}{l}\mathrm{CD}^{-} / \mathrm{CD}^{+} 6^{+} \\
\text {PBMCs }\end{array}$ & $\mathrm{N} / \mathrm{A}$ & No expansion & None & $\begin{array}{l}\text { High risk myeloid } \\
\text { malignancies }\end{array}$ & $\begin{array}{l}\text { Infusion of NKs post } \\
\text { haplo-HSCT }\end{array}$ & $\begin{array}{l}0.21-1.41 \times \\
10^{7} / \mathrm{kg}\end{array}$ & $N / D$ & $\begin{array}{l}\text { Well tolerated, increased donor } \\
\text { chimerism in } 2 / 5 \text { patients }\end{array}$ & (19) \\
\hline $\begin{array}{l}\mathrm{CD}^{-} / \mathrm{CD}^{+} 6^{+} \\
\text {PBMCs }\end{array}$ & None & 5 in 12 days (95\%) & IL-2 & $\begin{array}{l}\text { Multiple relapse ALL } \\
\text { and AML }\end{array}$ & $\begin{array}{l}\text { Repeat infusions of } \\
\text { activated NKs post-HSCT }\end{array}$ & $\begin{array}{l}8.9-29.5 \times \\
10^{6} / \mathrm{kg}\end{array}$ & $N / D$ & Well tolerated, no toxicity & (44) \\
\hline $\begin{array}{l}\mathrm{CD}^{-} \\
\text {PBMCs }\end{array}$ & None & No expansion & None & $\begin{array}{l}\text { Metastatic } \\
\text { melanoma, RCC, } \\
\text { refractory Hodgkin's, } \\
\text { and AML }\end{array}$ & $\begin{array}{l}\text { Infusion of NKs + IL-2 } \\
\text { after Lo-CY/mPred, FLU, } \\
\text { or Hi-CY/FLU }\end{array}$ & $\begin{array}{l}1 \times 10^{5}-2 \times \\
10^{7} / \mathrm{kg}\end{array}$ & $\begin{array}{l}\text { In vivo NK expansion } \\
\text { in } \mathrm{Hi}-\mathrm{Cy} / \text { Flu patients }\end{array}$ & $\begin{array}{l}\text { CR in } 5 / 19 \text { poor prognosis } \\
\text { patients }\end{array}$ & (10) \\
\hline $\begin{array}{l}\mathrm{CD}^{-} \\
\text {PBMCs }\end{array}$ & None & No expansion & $\mathrm{IL}-2$ & Myeloma & $\begin{array}{l}\text { Infusion of activated } \\
\text { NKs }+ \text { IL-2 after FLU/MEL } \\
\text { regimen and auto-PBSCT }\end{array}$ & $1.7 \times 10^{6} / \mathrm{kg}$ & $\begin{array}{l}\text { Donor cells persisted } \\
\text { and lost by day } 9-14\end{array}$ & CR in $50 \%$ patients & (45) \\
\hline PBMCs & None & $\begin{array}{l}1036 \text { in } 19 \text { days } \\
\text { (88\% viability) }\end{array}$ & $\begin{array}{l}\text { OKT3 } \\
\text { and IL-2 }\end{array}$ & $\begin{array}{l}\text { CRC, carcinoma and } \\
\text { B-CLL }\end{array}$ & $\begin{array}{l}\text { Infusion of activated } \\
\text { NKs + IL-2 after } \\
\text { haplo-HSCT }\end{array}$ & $\begin{array}{l}8.1-40.3 \times \\
10^{6} / \mathrm{kg}\end{array}$ & Multi-infusion trial & Minor response in 2 patients & (46) \\
\hline $\begin{array}{l}\mathrm{CD}^{-} / \mathrm{CD}^{2} 6^{+} \\
\text {PBMCs }\end{array}$ & None & No expansion & None & AML & $\begin{array}{l}\text { Infusion of NKs + IL-2 } \\
\text { after CY/FLU regimen }\end{array}$ & $\begin{array}{l}0.5-8.1 \times \\
10^{7} / \mathrm{kg}\end{array}$ & $\begin{array}{l}\text { Significant in vivo } \\
\text { expansion observed } \\
\text { at day } 14(5800 / \mathrm{mL})\end{array}$ & $100 \%$ EFS at 2 years & (47) \\
\hline $\begin{array}{l}\mathrm{CD}^{-} \\
\text {PBMCs }\end{array}$ & None & $\begin{array}{l}\text { No expansion } \\
(43 \pm 11 \%)\end{array}$ & None & Lymphoma & $\begin{array}{l}\text { Infusion of NKs + IL-2 } \\
\text { after RTX/CY/FLU }\end{array}$ & $\begin{array}{l}0.2-40 \times \\
10^{7} / \mathrm{kg}\end{array}$ & $\begin{array}{l}\text { NK cells not detected } \\
7 \text { days post infusion }\end{array}$ & $2 \mathrm{CR} / 2 \mathrm{PR}$ & (48) \\
\hline
\end{tabular}




\section{TABLE 1 | Continued}

\begin{tabular}{|c|c|c|c|c|c|c|c|c|c|}
\hline $\begin{array}{l}\text { Initial } \\
\text { population }\end{array}$ & $\begin{array}{l}\text { Feeder } \\
\text { cells }\end{array}$ & $\begin{array}{l}\text { Fold expansion } \\
\text { in vitro (purity) }\end{array}$ & $\begin{array}{l}\text { In vitro } \\
\text { cytokine } \\
\text { admin }\end{array}$ & Condition & $\begin{array}{l}\text { Treatment and } \\
\text { in vivo cytokine } \\
\text { admin }\end{array}$ & Dose & $\begin{array}{l}\text { In vivo } \\
\text { expansion }\end{array}$ & $\begin{array}{l}\text { Clinical } \\
\text { outcome }\end{array}$ & Reference \\
\hline $\begin{array}{l}\mathrm{CD}^{-} / \mathrm{CD}^{+} 6^{+} \\
\text {PBMCs }\end{array}$ & None & $\begin{array}{l}32-131.3 \text { in } \\
20-23 \text { days } \\
(82.7-99.6 \%)\end{array}$ & $\begin{array}{l}\mathrm{HC} \text { and } \\
\mathrm{IL}-15\end{array}$ & Advanced NSCLC & $\begin{array}{l}\text { Infusion of pre-activated } \\
\text { NKs }\end{array}$ & $\begin{array}{l}0.2-29 \times \\
10^{6} / \mathrm{kg}\end{array}$ & Multi-infusion trial & $\begin{array}{l}\text { PR in } 2 \text { patients best response } \\
\text { with most infusions }\end{array}$ & (49) \\
\hline $\begin{array}{l}\mathrm{CD}^{+} 6^{+} \\
\text {selected } \\
\text { PBMCs }\end{array}$ & None & No expansion & None & AML & $\begin{array}{l}\text { Infusion of NKs + IL-2 } \\
\text { after CY/FLU regimen }\end{array}$ & $\begin{array}{l}1.11-5.0 \times \\
10^{6} / \mathrm{kg}\end{array}$ & $\begin{array}{l}\text { Donor NKs detected } \\
\text { up to } 17 \text { days post } \\
\text { first infusion }\end{array}$ & CR 6/13 patient & (50) \\
\hline $\begin{array}{l}\mathrm{CD}^{-} / \mathrm{CD}^{+} 6^{+} \\
\text {PBMCs }\end{array}$ & None & No expansion & $\begin{array}{l}\text { IL-2 for } \\
\text { half of } \\
\text { patients }\end{array}$ & $\begin{array}{l}\text { AML, ALL, } \\
\text { neuroblastoma, and } \\
\text { RMS }\end{array}$ & $\begin{array}{l}\text { Multiple infusions of } \\
\text { pre-activated and resting } \\
\text { NKs after haplo-HSCT }\end{array}$ & $\begin{array}{l}6-45.1 \times \\
10^{6} / \mathrm{kg}\end{array}$ & $\begin{array}{l}\text { NK cells detected at } \\
24 \mathrm{~h}\end{array}$ & $\begin{array}{l}\text { Two patients with } \\
\text { neuroblastoma alive at } 2 \text { years }\end{array}$ & (51) \\
\hline $\begin{array}{l}\mathrm{CD}^{-} \\
\text {PBMCs }\end{array}$ & None & $\begin{array}{l}\text { No expansion } \\
\text { (70\% viability) }\end{array}$ & IL-2 & $\begin{array}{l}\text { Breast and ovarian } \\
\text { carcinoma }\end{array}$ & $\begin{array}{l}\text { Infusion of pre-activated } \\
\text { NKs + IL-2 after CY/FLU } \\
\text { with/without TBI }\end{array}$ & $\begin{array}{l}8.33 \times \\
10^{6}-3.94 \times \\
10^{7} / \mathrm{kg}\end{array}$ & $\begin{array}{l}\text { No eligible patients } \\
\text { met predefined } \\
\text { criterion for } \\
\text { successful in vivo } \\
\text { expansion }\end{array}$ & $\begin{array}{l}\text { TBI improved longevity of NK } \\
\text { engraftment }\end{array}$ & (52) \\
\hline $\begin{array}{l}\mathrm{CD}^{+} 6^{+/} \\
\mathrm{CD}^{-} \\
\text {PBMCs }\end{array}$ & None & No expansion & None & $\begin{array}{l}\text { Leukemia and } \\
\text { malignant solid } \\
\text { tumors }\end{array}$ & $\begin{array}{l}\text { Multiple NK infusions after } \\
\text { ATG/OKT3 and } \\
\text { hapol-HSCT }\end{array}$ & $\begin{array}{l}0.3-3.8 \times \\
10^{7} / \mathrm{kg}\end{array}$ & N/D & No significant clinical response & (53) \\
\hline $\begin{array}{l}\mathrm{CD} 56^{+} / \mathrm{CD} 3 / \\
\mathrm{CD}^{-} 9^{-} \\
\text {PBMCs }\end{array}$ & None & $\begin{array}{l}\text { No expansion } \\
(53 \%)\end{array}$ & IL-2 & $\begin{array}{l}\text { Relapsed/primary } \\
\text { AML }\end{array}$ & $\begin{array}{l}\text { Infusion pre-activated NKs } \\
\text { after IL-2DT }\end{array}$ & $\begin{array}{l}2.6 \pm 1.5 \times \\
10^{7} / \mathrm{kg}\end{array}$ & $\begin{array}{l}\text { In vivo expansion } \\
\text { enhanced with } \\
\text { T-REG depletion }\end{array}$ & Well tolerated, no toxicity & (11) \\
\hline $\begin{array}{l}\mathrm{CD}_{56}{ }^{+} \mathrm{CD}^{-} \\
\text {PBMCs }\end{array}$ & $\begin{array}{l}4-1 \mathrm{BBL}^{+} \mathrm{IL}- \\
15 \mathrm{R} \alpha^{+} \\
\text {aAPCs }\end{array}$ & $\begin{array}{l}\text { 9-11 days } \\
(>90 \%)\end{array}$ & IL-15 & $\begin{array}{l}\text { EWS, DSRCT, and } \\
\text { RMS }\end{array}$ & CY/FLU/MEL/G-CSF & $1 \times 10^{5} / \mathrm{kg}$ & Multi-infusion trial & $\begin{array}{l}\text { 5/9 patients experienced acute } \\
\text { GVHD }\end{array}$ & (25) \\
\hline \multicolumn{10}{|l|}{ CELL LINES } \\
\hline $\begin{array}{l}\text { NK92 cell } \\
\text { line }\end{array}$ & None & $\begin{array}{l}>200 \text { in } \\
15-17 \text { days }\end{array}$ & IL-2 & $\begin{array}{l}\text { RCC and malignant } \\
\text { melanoma }\end{array}$ & $\begin{array}{l}\text { Infusion of ex vivo } \\
\text { expanded NK-92 cells }\end{array}$ & $\begin{array}{l}\text { Up to } \\
3 \times 10^{9} / \mathrm{m}^{2}\end{array}$ & Multi-infusion trial & $\begin{array}{l}\text { Well-tolerated possible } \\
\text { response in } 2 \text { patients }\end{array}$ & (27) \\
\hline $\begin{array}{l}\text { NK92 cell } \\
\text { line }\end{array}$ & None & 2 in $32 \mathrm{~h}$ & IL-2 & $\begin{array}{l}\text { Solid tumors, } \\
\text { sarcomas, } \\
\text { leukemias, and } \\
\text { lymphoma }\end{array}$ & $\begin{array}{l}\text { Infusion of ex vivo } \\
\text { expanded NK-92 cells }\end{array}$ & $\begin{array}{l}\text { Up to } \\
1 \times 10^{10} / \mathrm{m}^{2}\end{array}$ & $\begin{array}{l}\text { Persist in circulation } \\
\text { up to } 48 \mathrm{~h}\end{array}$ & $\begin{array}{l}\text { Well-tolerated possible } \\
\text { response in lung cancer } \\
\text { patients }\end{array}$ & (28) \\
\hline $\begin{array}{l}\mathrm{CD}^{+} 6^{+} \mathrm{CD}^{-} \\
\text {PBMCs }\end{array}$ & None & $\begin{array}{l}5 \text { in } 14 \text { days } \\
(>95 \%)\end{array}$ & IL-2 & $\begin{array}{l}\text { Multiple relapsed } \\
\text { neuroblastoma }\end{array}$ & $\begin{array}{l}\text { Infusion pre-activated NK } \\
\text { cells }\end{array}$ & $\begin{array}{l}7.8-45.1 \times \\
10^{6} / \mathrm{kg}\end{array}$ & No clear expansion & No toxicity observed & (54) \\
\hline
\end{tabular}

ALL, acute lymphoblastic lymphoma; AML, acute myeloid leukemia; ATG, anti-thymocyte globulin; B-CLL, B cell chronic lymphocyte leukemia; BOR, bortezomib; CLL, chronic lymphocyte leukemia; CR, complete response; CRC, colorectal carcinoma; CY, cyclophosphamide; DSRCT, desmoplastic small round cell tumor; EWS, Ewing sarcoma; FLU, fludarabine; G-CSF, granulocyte colony stimulating factor; HC, hydrocortisone; MEL, melphalan; MM, multiple myeloma; mPred, methy/prednisolone; MRC, metastatic renal carcinoma; N/D, not determined; NSCLC, non-small cell lung carcinoma; PEN, pentostatin; PR, partial response; RCC, renal cell carcinoma; RMS, rhabdomyosarcoma; RTX, rituximab; TBI, total body irradiation. 


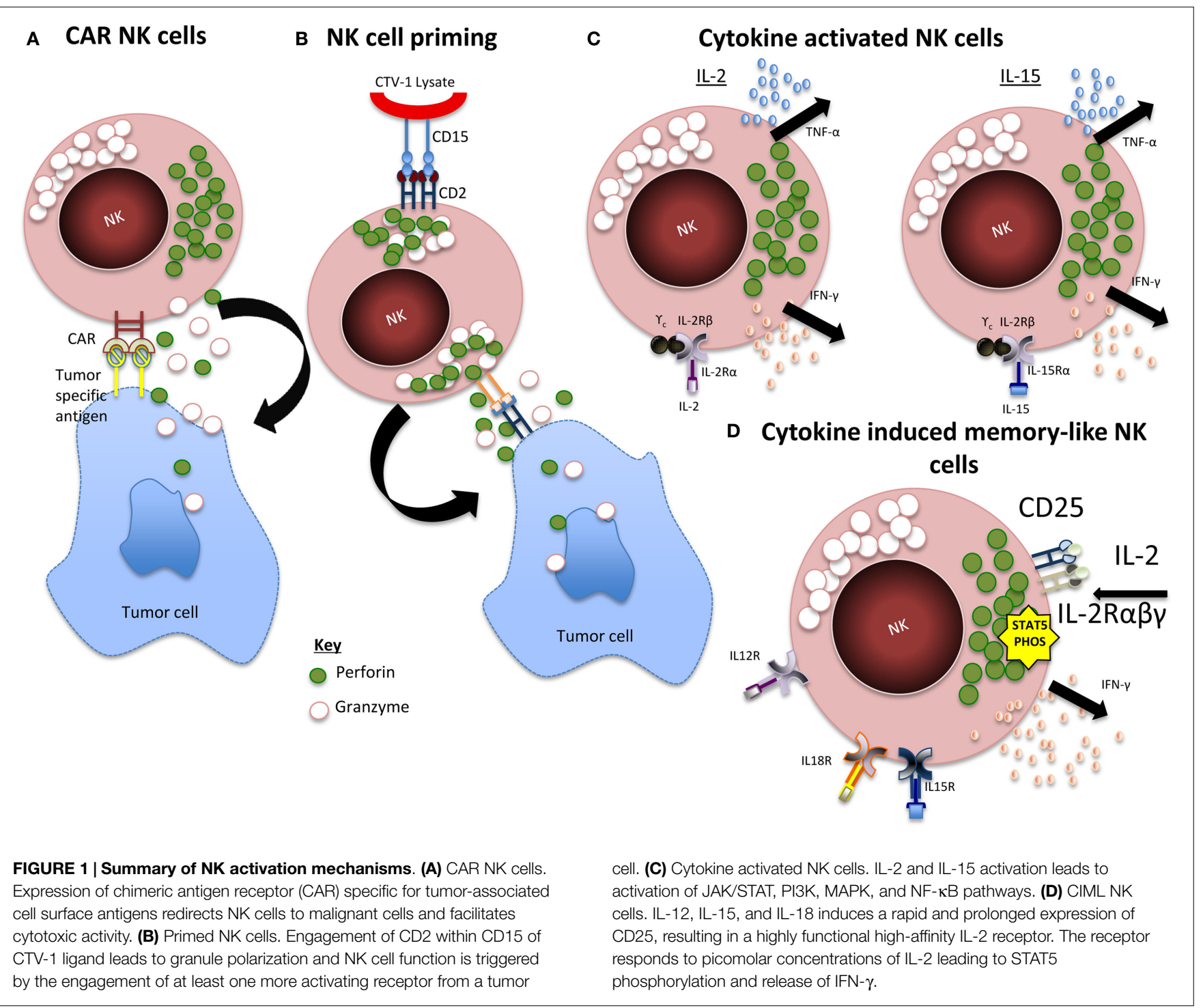

toxicity; however, its reduced half life may suggest the need for more frequent dosing in therapeutic applications (59).

A method to avoid such life threatening conditions through in vivo administration of cytokines is expanding or stimulating the cells in vitro. NK cells have always been considered a member of the innate immune system incapable of producing memory. However, in 2006, it was first observed that NK cells could mediate a long-lived antigen-specific adaptive response independently of other lymphocytes (60). Sun and colleagues (61) later identified an immunological memory in NK cells from MCMV infected mice and it was demonstrated that NK cells pre-activated with IL-12 and IL-18 infused into a naive host and later re-stimulated showed enhanced IFN-y production (62). This model was later transferred to an in vitro model stimulating human cells showing the same results (63). This improvement in cytokine production offers the potential for enhanced GvL effect and a clinical trial is currently in progress targeting relapsed and refractory AML (NCT01898793).

It has been reported that resting NK cells require a two-stage activation process known as "priming" and "triggering" (64). This states that tumors resistant to NK cell killing evade lysis by failing to prime the cell; however, Mark Lowdells group were able to identify a cell line, which could prime the cell without triggering cytokine production or cytolytic activity. This led to the development of an NK cell priming technique that readied the cells for killing, which was still maintained post cryopreservation (65). Primed NK cells from patients with multiple myeloma have also been proven to kill NK cell resistant malignant plasma cells (66). Preliminary data from an ongoing transitional phase I/II clinical trial showed that without cytokine administration primed NK cells from HLA haploidentical-related donors can persist in vivo with no toxic effects (67).

\section{Genetic Engineering}

Although currently restricted to pre-clinical models the use of chimeric antigen receptor (CAR)-expressing NK cells has the potential to offer enhanced effector cell function of increased specificity. Anti-CD19 CAR T cells have effectively demonstrated their ability to induce long-term remission in patients with $\mathrm{B}$ cell malignancies (68). However, concern associated with CAR 
T cell therapy extends to GvHD, on target/off tumor effects, and tumor lysis syndrome. By contrast, allogeneic CAR-engineered NK cells are expected to induce anti-tumor effects and dissipate after a few days (23). As previously reviewed by Glienke et al. in 2015, current work in the field has focused mainly on targeting CD19 and CD20, however, CARs, which target CS1 and CD138 for multiple myeloma, GD2 and CD244 for neuroblastoma, HER2 for epithelial carcinomas, and GPA7 for melanoma, are also beginning to indicate promising results.

\section{Immune Escape Mechanisms}

Not all tumors are susceptible to NK cell mediated killing, as some cancer cells have developed the ability to escape detection by the immune system. Mechanisms that regulate the evasion of tumor cells by NK cells extends to the down-regulation of activating receptor ligands for NKG2D (69), the production of soluble stressinduced ligands, such as MICA, which degrades NKG2D leading to NK cell inhibition (70) and the release of suppressive cytokines such as IL-10 and TGF- $\beta$ (71). Some success has been seen by NK cell immunotherapy targeting hematological malignancies; however, this has not been transferred to solid tumors. This could be a result of the increased concentration of immunosuppressive cytokines and ligands around a tumor mass, method to overcome such escape mechanisms could provide further potential for NK cells to not only target hematological malignancies but also solid tumors.

\section{Concluding Remarks}

Natural killer cell immunotherapy has been a promising option for providing specialized and target specific treatment for a therapy

\section{References}

1. Cooper MA. Human natural killer cells: a unique innate immunoregulatory role for the CD56bright subset. Blood (2001) 97:3146-51. doi:10.1182/blood.V97. 10.3146

2. Karre K, Ljunggren HG, Piontek G, Kiessling R. Selective rejection of H-2deficient lymphoma variants suggests alternative immune defence strategy. Nature (1986) 319:675-8. doi:10.1038/319675a0

3. Karre K. NK cells, MHC class I molecules and the missing self. Scand J Immunol (2002) 55:221-8. doi:10.1046/j.1365-3083.2002.01053.x

4. Di Santo JP. Natural killer cell developmental pathways: a question of balance. Annu Rev Immunol (2006) 24:257-86. doi:10.1146/annurev.immunol.24. 021605.090700

5. Imai K, Matsuyama S, Miyake S, Suga K, Nakachi K. Natural cytotoxic activity of peripheral-blood lymphocytes and cancer incidence: an 11-year followup study of a general population. Lancet (2000) 356:1795-9. doi:10.1016/ S0140-6736(00)03231-1

6. Ljunggren HG, Malmberg KJ. Prospects for the use of NK cells in immunotherapy of human cancer. Nat Rev Immunol (2007) 7:329-39. doi:10.1038/nri2073

7. Burns LJ, Weisdorf DJ, Defor TE, Vesole DH, Repka TL, Blazar BR, et al. IL2-based immunotherapy after autologous transplantation for lymphoma and breast cancer induces immune activation and cytokine release: a phase I/II trial. Bone Marrow Transplant (2003) 32:177-86. doi:10.1038/sj.bmt.1704086

8. Ruggeri L, Capanni M, Urbani E, Perruccio K, Shlomchik WD, Tosti A, et al. Effectiveness of donor natural killer cell alloreactivity in mismatched hematopoietic transplants. Science (2002) 295:2097-100. doi:10.1126/science. 1068440

9. Ruggeri L, Mancusi A, Capanni M, Martelli MF, Velardi A. Exploitation of alloreactive NK cells in adoptive immunotherapy of cancer. Curr Opin Immunol (2005) 17:211-7. doi:10.1016/j.coi.2005.01.007 in its own right and as a supportive one in infection or transplantation. Although some mechanisms of NK cell biology are yet to be elucidated as we make progress in the field an effective clinical NK cell immunotherapy will become more achievable. A standard clinical regimen is still to be elucidated and obstacles such as cell dose, activation status, method of expansion, drug complement, and source are still to be determined.

It has always been thought that high numbers of NK cells are necessary for a successful clinical product. However, numerous groups have managed to successfully generate high numbers of functional NK cells in vitro although the lack of clinical effect and significant cost implications cannot be ignored. The highcell number requirement is likely to be the result of a "success in numbers" approach with there being a significant loss of cells through in vivo targeting and just a small sub-population of effector cells that will target the tumor. Perhaps work should therefore be refocused on the infusion of a small population of cells with optimum pre-activation status that will traffic to the tumor site and would not be suppressed by tumor evasion mechanisms. This is a significant goal to achieve considering the variety of NK cell populations occurring naturally in the body. However, the absence of a labor-intensive long-term culture system would mean this method would pose significantly reduced cost implications. Once techniques have been optimized and streamlined there would therefore be a greater possibility of NK cell immunotherapy being routinely adopted as a clinical therapy in the future.

\section{Acknowledgments}

This work was funded and supported by Anthony Nolan.

10. Miller JS, Soignier Y, Panoskaltsis-Mortari A, Mcnearney SA, Yun GH, Fautsch SK, et al. Successful adoptive transfer and in vivo expansion of human haploidentical NK cells in patients with cancer. Blood (2005) 105:3051-7. doi:10. 1182/blood-2004-07-2974

11. Bachanova V, Cooley S, Defor TE, Verneris MR, Zhang B, Mckenna DH, et al Clearance of acute myeloid leukemia by haploidentical natural killer cells is improved using IL-2 diphtheria toxin fusion protein. Blood (2014) 123:3855-63. doi:10.1182/blood-2013-10-532531

12. Ishikawa E, Tsuboi K, Saijo K, Harada H, Takano S, Nose T, et al. Autologous natural killer cell therapy for human recurrent malignant glioma. Anticancer Res (2004) 24(3b):1861-71.

13. Tarek N, Le Luduec JB, Gallagher MM, Zheng J, Venstrom JM, Chamberlain E, et al. Unlicensed NK cells target neuroblastoma following anti-GD2 antibody treatment. J Clin Invest (2012) 122:3260-70. doi:10.1172/JCI62749

14. Bacigalupo A, Ballen K, Rizzo D, Giralt S, Lazarus H, Ho V, et al. Defining the intensity of conditioning regimens: working definitions. Biol Blood Marrow Transplant (2009) 15:1628-33. doi:10.1016/j.bbmt.2009.07.004

15. Morgan GJ, Davies FE. Role of thalidomide in the treatment of patients with multiple myeloma. Crit Rev Oncol Hematol (2013) 88(Suppl 1):S14-22. doi:10. 1016/j.critrevonc.2013.05.012

16. Davies F, Baz R. Lenalidomide mode of action: linking bench and clinical findings. Blood Rev (2010) 24(Suppl 1):S13-9. doi:10.1016/S0268-960X(10) 70004-7

17. Semeraro M, Vacchelli E, Eggermont A, Galon J, Zitvogel L, Kroemer G, et al. Trial Watch: lenalidomide-based immunochemotherapy. Oncoimmunology (2013) 2:e26494. doi:10.4161/onci.26494

18. Lundqvist A, Su S, Rao S, Childs R. Cutting edge: bortezomib-treated tumors sensitized to NK cell apoptosis paradoxically acquire resistance to antigen-specific T cells. J Immunol (2010) 184:1139-42. doi:10.4049/jimmunol. 0902856 
19. Passweg JR, Tichelli A, Meyer-Monard S, Heim D, Stern M, Kuhne T, et al. Purified donor NK-lymphocyte infusion to consolidate engraftment after haploidentical stem cell transplantation. Leukemia (2004) 18:1835-8. doi:10.1038/ sj.leu. 2403524

20. Choi I, Yoon SR, Park SY, Kim H, Jung SJ, Jang YJ, et al. Donor-derived natural killer cells infused after human leukocyte antigen-haploidentical hematopoietic cell transplantation: a dose-escalation study. Biol Blood Marrow Transplant (2014) 20:696-704. doi:10.1016/j.bbmt.2014.01.031

21. Cheng M, Chen Y, Xiao W, Sun R, Tian Z. NK cell-based immunotherapy for malignant diseases. Cell Mol Immunol (2013) 10:230-52. doi:10.1038/cmi. 2013.10

22. Koehl U, Brehm C, Huenecke S, Zimmermann SY, Kloess S, Bremm M, et al. Clinical grade purification and expansion of NK cell products for an optimized manufacturing protocol. Front Oncol (2013) 3:118. doi:10.3389/fonc. 2013.00118

23. Klingemann H. Challenges of cancer therapy with natural killer cells. Cytotherapy (2015) 17(3):245-9. doi:10.1016/j.jcyt.2014.09.007

24. Fujisaki H, Kakuda H, Shimasaki N, Imai C, Ma J, Lockey T, et al. Expansion of highly cytotoxic human natural killer cells for cancer cell therapy. Cancer Res (2009) 69:4010-7. doi:10.1158/0008-5472.CAN-08-3712

25. Shah NN, Baird K, Delbrook CP, Fleisher TA, Kohler ME, Rampertaap S, et al. Acute GVHD in patients receiving IL-15/4-1BBL activated NK cells following T cell depleted stem cell transplantation. Blood (2014) 125(5):784-92. doi:10. 1182/blood-2014-07-592881

26. Klingemann HG, Wong E, Maki G. A cytotoxic NK-cell line (NK-92) for ex vivo purging of leukemia from blood. Biol Blood Marrow Transplant (1996) 2:68-75.

27. Arai S, Meagher R, Swearingen M, Myint H, Rich E, Martinson J, et al. Infusion of the allogeneic cell line NK-92 in patients with advanced renal cell cancer or melanoma: a phase I trial. Cytotherapy (2008) 10:625-32. doi:10.1080/ 14653240802301872

28. Tonn T, Schwabe D, Klingemann HG, Becker S, Esser R, Koehl U, et al. Treatment of patients with advanced cancer with the natural killer cell line NK-92. Cytotherapy (2013) 15:1563-70. doi:10.1016/j.jcyt.2013.06.017

29. Woll PS, Grzywacz B, Tian X, Marcus RK, Knorr DA, Verneris MR, et al. Human embryonic stem cells differentiate into a homogeneous population of natural killer cells with potent in vivo antitumor activity. Blood (2009) 113:6094-101. doi:10.1182/blood-2008-06-165225

30. Miller JS, Alley KA, Mcglave P. Differentiation of natural killer (NK) cells from human primitive marrow progenitors in a stroma-based long-term culture system: identification of a CD34+7+ NK progenitor. Blood (1994) 83:2594-601.

31. Shibuya A, Nagayoshi K, Nakamura K, Nakauchi H. Lymphokine requirement for the generation of natural killer cells from CD34+ hematopoietic progenitor cells. Blood (1995) 85:3538-46.

32. Knorr DA, Ni Z, Hermanson D, Hexum MK, Bendzick L, Cooper LJ, et al. Clinical-scale derivation of natural killer cells from human pluripotent stem cells for cancer therapy. Stem Cells Transl Med (2013) 2:274-83. doi:10.5966/ sctm.2012-0084

33. Eguizabal C, Zenarruzabeitia O, Monge J, Santos S, Vesga MA, Maruri N, et al. Natural killer cells for cancer immunotherapy: pluripotent stem cells-derived NK cells as an immunotherapeutic perspective. Front Immunol (2014) 5:439. doi:10.3389/fimmu.2014.00439

34. Yoon SR, Lee YS, Yang SH, Ahn KH, Lee JH, Lee JH, et al. Generation of donor natural killer cells from CD34(+) progenitor cells and subsequent infusion after HLA-mismatched allogeneic hematopoietic cell transplantation: a feasibility study. Bone Marrow Transplant (2010) 45:1038-46. doi:10.1038/bmt. 2009.304

35. Spanholtz J, Tordoir M, Eissens D, Preijers F, Van Der Meer A, Joosten I, et al. High log-scale expansion of functional human natural killer cells from umbilical cord blood CD34-positive cells for adoptive cancer immunotherapy. PLoS One (2010) 5:e9221. doi:10.1371/journal.pone.0009221

36. Grzywacz B, Kataria N, Sikora M, Oostendorp RA, Dzierzak EA, Blazar BR, et al. Coordinated acquisition of inhibitory and activating receptors and functional properties by developing human natural killer cells. Blood (2006) 108:3824-33. doi:10.1182/blood-2006-04-020198

37. Luevano M, Domogala A, Blundell M, Jackson N, Pedroza-Pacheco I, Derniame $S$, et al. Frozen cord blood hematopoietic stem cells differentiate into higher numbers of functional natural killer cells in vitro than mobilized hematopoietic stem cells or freshly isolated cord blood hematopoietic stem cells. PLoS One (2014) 9:e87086. doi:10.1371/journal.pone.0087086
38. Escudier B, Farace F, Angevin E, Charpentier F, Nitenberg G, Triebel F, et al. Immunotherapy with interleukin-2 (IL2) and lymphokine-activated natural killer cells: improvement of clinical responses in metastatic renal cell carcinoma patients previously treated with IL2. Eur J Cancer (1994) 30a:1078-83. doi:10. 1016/0959-8049(94)90460-X

39. Krause SW, Gastpar R, Andreesen R, Gross C, Ullrich H, Thonigs G, et al. Treatment of colon and lung cancer patients with ex vivo heat shock protein 70-peptide-activated, autologous natural killer cells: a clinical phase i trial. Clin Cancer Res (2004) 10:3699-707. doi:10.1158/1078-0432.CCR-03-0683

40. Motohashi S, Ishikawa A, Ishikawa E, Otsuji M, Iizasa T, Hanaoka H, et al. A phase I study of in vitro expanded natural killer T cells in patients with advanced and recurrent non-small cell lung cancer. Clin Cancer Res (2006) 12:6079-86. doi:10.1158/1078-0432.CCR-06-0114

41. Lundqvist A, Berg M, Smith A, Childs RW. Bortezomib treatment to potentiate the anti-tumor immunity of ex-vivo expanded adoptively infused autologous natural killer cells. J Cancer (2011) 2:383-5. doi:10.7150/jca.2.383

42. Parkhurst MR, Riley JP, Dudley ME, Rosenberg SA. Adoptive transfer of autologous natural killer cells leads to high levels of circulating natural killer cells but does not mediate tumor regression. Clin Cancer Res (2011) 17:6287-97. doi:10.1158/1078-0432.CCR-11-1347

43. Szmania S, Lapteva N, Garg T, Greenway A, Lingo J, Nair B, et al. Ex vivoexpanded natural killer cells demonstrate robust proliferation in vivo in highrisk relapsed multiple myeloma patients. J Immunother (2014) 38(1):24-36. doi:10.1097/CJI.0000000000000059

44. Koehl U, Sorensen J, Esser R, Zimmermann S, Gruttner HP, Tonn T, et al. IL2 activated NK cell immunotherapy of three children after haploidentical stem cell transplantation. Blood Cells Mol Dis (2004) 33:261-6. doi:10.1016/j.bcmd. 2004.08.013

45. Shi J, Tricot G, Szmania S, Rosen N, Garg TK, Malaviarachchi PA, et al. Infusion of haplo-identical killer immunoglobulin-like receptor ligand mismatched NK cells for relapsed myeloma in the setting of autologous stem cell transplantation. Br J Haematol (2008) 143:641-53. doi:10.1111/j.1365-2141. 2008.07340.x

46. Barkholt L, Alici E, Conrad R, Sutlu T, Gilljam M, Stellan B, et al. Safety analysis of ex vivo-expanded NK and NK-like T cells administered to cancer patients: a phase I clinical study. Immunotherapy (2009) 1:753-64. doi:10.2217/ imt.09.47

47. Rubnitz JE, Inaba H, Ribeiro RC, Pounds S, Rooney B, Bell T, et al. NKAML: a pilot study to determine the safety and feasibility of haploidentical natural killer cell transplantation in childhood acute myeloid leukemia. J Clin Oncol (2010) 28:955-9. doi:10.1200/JCO.2009.24.4590

48. Bachanova V, Burns LJ, Mckenna DH, Curtsinger J, Panoskaltsis-Mortari A, Lindgren BR, et al. Allogeneic natural killer cells for refractory lymphoma. Cancer Immunol Immunother (2010) 59:1739-44. doi:10.1007/s00262-010-0896-Z

49. Iliopoulou EG, Kountourakis P, Karamouzis MV, Doufexis D, Ardavanis A, Baxevanis $\mathrm{CN}$, et al. A phase I trial of adoptive transfer of allogeneic natural killer cells in patients with advanced non-small cell lung cancer. Cancer Immunol Immunother (2010) 59:1781-9. doi:10.1007/s00262-010-0904-3

50. Curti A, Ruggeri L, D’Addio A, Bontadini A, Dan E, Motta MR, et al. Successful transfer of alloreactive haploidentical KIR ligand-mismatched natural killer cells after infusion in elderly high risk acute myeloid leukemia patients. Blood (2011) 118:3273-9. doi:10.1182/blood-2011-01-329508

51. Brehm C, Huenecke S, Quaiser A, Esser R, Bremm M, Kloess S, et al. IL-2 stimulated but not unstimulated NK cells induce selective disappearance of peripheral blood cells: concomitant results to a phase I/II study. PLoS One (2011) 6:e27351. doi:10.1371/journal.pone.0027351

52. Geller MA, Cooley S, Judson PL, Ghebre R, Carson LF, Argenta PA, et al. A phase II study of allogeneic natural killer cell therapy to treat patients with recurrent ovarian and breast cancer. Cytotherapy (2011) 13:98-107. doi:10 3109/14653249.2010.515582

53. Stern M, Passweg JR, Meyer-Monard S, Esser R, Tonn T, Soerensen J, et al. Preemptive immunotherapy with purified natural killer cells after haploidentical SCT: a prospective phase II study in two centers. Bone Marrow Transplant (2013) 48:433-8. doi:10.1038/bmt.2012.162

54. Huenecke S, Zimmermann SY, Kloess S, Esser R, Brinkmann A, Tramsen $\mathrm{L}$, et al. IL-2-driven regulation of NK cell receptors with regard to the distribution of CD16+ and CD16- subpopulations and in vivo influence after haploidentical NK cell infusion. J Immunother (2010) 33:200-10. doi:10.1097/ CJI.0b013e3181bb46f7 
55. Trinchieri G, Matsumoto-Kobayashi M, Clark SC, Seehra J, London L, Perussia B. Response of resting human peripheral blood natural killer cells to interleukin 2. J Exp Med (1984) 160:1147-69. doi:10.1084/jem.160.4.1147

56. Carson WE, Fehniger TA, Haldar S, Eckhert K, Lindemann MJ, Lai CF, et al. A potential role for interleukin-15 in the regulation of human natural killer cell survival. J Clin Invest (1997) 99:937-43. doi:10.1172/JCI119258

57. Krieg C, Letourneau S, Pantaleo G, Boyman O. Improved IL-2 immunotherapy by selective stimulation of IL-2 receptors on lymphocytes and endothelial cells. Proc Natl Acad Sci U S A (2010) 107:11906-11. doi:10.1073/pnas.1002569107

58. Ahmadzadeh M, Rosenberg SA. IL-2 administration increases CD4+ CD25(hi) Foxp3+ regulatory T cells in cancer patients. Blood (2006) 107:2409-14. doi:10. 1182/blood-2005-06-2399

59. Berger C, Berger M, Hackman RC, Gough M, Elliott C, Jensen MC, et al. Safety and immunologic effects of IL-15 administration in nonhuman primates. Blood (2009) 114:2417-26. doi:10.1182/blood-2008-12-189266

60. O'Leary JG, Goodarzi M, Drayton DL, Von Andrian UH. T cell- and B cellindependent adaptive immunity mediated by natural killer cells. Nat Immunol (2006) 7:507-16. doi:10.1038/ni1332

61. Sun JC, Beilke JN, Lanier LL. Adaptive immune features of natural killer cells. Nature (2009) 457:557-61. doi:10.1038/nature07665

62. Cooper MA, Elliott JM, Keyel PA, Yang L, Carrero JA, Yokoyama WM. Cytokine-induced memory-like natural killer cells. Proc Natl Acad Sci U S A (2009) 106:1915-9. doi:10.1073/pnas.0813192106

63. Romee R, Schneider SE, Leong JW, Chase JM, Keppel CR, Sullivan RP, et al. Cytokine activation induces human memory-like NK cells. Blood (2012) 120:4751-60. doi:10.1182/blood-2012-04-419283

64. North J, Bakhsh I, Marden C, Pittman H, Addison E, Navarrete C, et al. Tumorprimed human natural killer cells lyse NK-resistant tumor targets: evidence of a two-stage process in resting NK cell activation. J Immunol (2007) 178:85-94. doi:10.4049/jimmunol.178.1.85

65. Sabry M, Tsirogianni M, Bakhsh IA, North J, Sivakumaran J, Giannopoulos K, et al. Leukemic priming of resting NK cells is killer Ig-like receptor independent but requires CD15-mediated CD2 ligation and natural cytotoxicity receptors. $J$ Immunol (2011) 187:6227-34. doi:10.4049/jimmunol.1101640

66. Katodritou E, Terpos E, North J, Kottaridis P, Verrou E, Gastari V, et al. Tumorprimed natural killer cells from patients with multiple myeloma lyse autologous, NK-resistant, bone marrow-derived malignant plasma cells. Am J Hemato (2011) 86:967-73. doi:10.1002/ajh.22163

67. Fehniger TA, Stuart RK, Cooley SA, Miller JS, Curtsinger J, Hillman TM, et al. Preliminary results of a phase $1 / 2$ clinical trial of Cndo-109-activated allogeneic natural killer cells in high risk acute myelogenous leukemia patients in first complete remission. Blood (2014) 124:2320-2320.

68. Grupp SA, Kalos M, Barrett D, Aplenc R, Porter DL, Rheingold SR, et al. Chimeric antigen receptor-modified T cells for acute lymphoid leukemia. $N$ Engl J Med (2013) 368:1509-18. doi:10.1056/NEJMoa1215134

69. Raffaghello L, Prigione I, Airoldi I, Camoriano M, Levreri I, Gambini C, et al. Downregulation and/or release of NKG2D ligands as immune evasion strategy of human neuroblastoma. Neoplasia (2004) 6:558-68. doi:10.1593/neo. 04316

70. Groh V, Wu J, Yee C, Spies T. Tumour-derived soluble MIC ligands impair expression of NKG2D and T-cell activation. Nature (2002) 419:734-8. doi:10. 1038/nature01112

71. Beissert S, Schwarz A, Schwarz T. Regulatory T cells. J Invest Dermatol (2006) 126:15-24. doi:10.1038/sj.jid.5700004

Conflict of Interest Statement: The authors declare that the research was conducted in the absence of any commercial or financial relationships that could be construed as a potential conflict of interest.

Copyright (C) 2015 Domogala, Madrigal and Saudemont. This is an open-access article distributed under the terms of the Creative Commons Attribution License (CC BY). The use, distribution or reproduction in other forums is permitted, provided the original author(s) or licensor are credited and that the original publication in this journal is cited, in accordance with accepted academic practice. No use, distribution or reproduction is permitted which does not comply with these terms. 